\title{
Simulation of Improved Fourth-Order Colpitts Oscillator
}

\author{
http://dx.doi.org/10.3991/ijoe.v9i3.2830 \\ Xiaohua QIAO, Yuxia SUN, Hongqin ZHANG and Yaping XU \\ School of Electrical and Information Engineering, Jiangsu University of Technology, Changzhou, China
}

\begin{abstract}
A RLC parallel resonance circuit was built on the basis of the original fourth-order Colpitts oscillator. An improved Colpitts oscillator was given. The Lyapunov exponent spectrum, bifurcation diagram and phase trajectories of chaotic attractors were calculated by control theory and numerical methods. Analysis shows that the change of resistance value $R$ of the RLC parallel resonance circuit to control Colpitts oscillator can obtain better dynamic characteristics than the original fourth-order Colpitts oscillator, and the control circuit implementation is very simple. More interestingly, it is found that the bifurcation behavior of the new system has the Fengenbaum first constant in the logarithm law. The experimental circuit of the new system was designed. The experimental results and theoretical analysis have a good consistency, indicating that this control scheme is of feasibility and better practicability.
\end{abstract}

Index Terms-Colpitts oscillator, Chaotic signals, Logarithmic Fengenbaum constant, Circuit implementation

\section{INTRODUCTION}

Chaotic signal, with the continuous noise-like powerspectral characteristics in the frequency domain, has been used in spread spectrum communications, secure communications and so on [1-3], which has become a hot topic in the chaos applied research in recent years. The core technology in this chaotic communication system is the design of broadband chaotic signal generator. The study history can be traced back to the analysis and experiment of Van Der Pol oscillator in 1927 [4]. Chua's circuit appeared and became the typical circuit of chaotic oscillator in the eighties of last century. The more use of the operational amplifier to achieve Chua's diode by this circuit limited the signal frequency of oscillator to the low frequency range. Therefore, the circuit is difficult to use in a high-frequency communication system [5].

Kennedy put forward a third-order Colpitts chaotic oscillator on the basis of the three-point sine wave oscillator of capacitance based on nonlinear oscillation theory in 1994. The research results of experimental circuit were also given [6]. Subsequently, Oscar, etc. further gave the more complete theoretical analysis and design results of third-order Colpitts chaotic oscillator which provide the theoretical and experimental bases for the in-depth study of the Colpitts chaotic oscillator [7-8]. $\mathrm{Yu}$ SiMin connected a variable capacitor in parallel with the inductor of the third-order Colpitts chaotic oscillator in 2008 and thus proposed a fourth-order Colpitts chaotic oscillator [9]. Then, the effect of the change in the variable capacitance value on the dynamics of Colpitts chaotic oscillator was verified and discussed through numerical analysis and experiment. There exist multiple period windows in the change interval $(\mathrm{C} 3: 0 \sim 5 \mathrm{nF})$ of this circuit variable capacitance. The controllability of slight variation of capacitance value is poor for the variable capacitor elements. In addition, the parasitic capacitance and other factors also have effect on it. Thereby, the requirements for circuit implementation are higher. Therefore, the circuit is difficult to apply to the communication systems and other fields of information engineering.

An improved fourth-order Colpitts oscillator was constructed on the basis of the fourth-order Colpitts oscillator put forward by $\mathrm{Yu}$ SiMin by connecting a potentiometer with an appropriate change in resistance in parallel with the LC parallel loop in this study. An analysis is on the basis of circuit modeling, the numerical calculation of the equilibrium point of the system, Lyapunov exponent spectrum, bifurcation diagram and phase trajectory. It found that the output chaotic signal of system is of stronger robustness in the larger range of change in high resistance of the potentiometer. Robust sine wave signal with a single or multiple cycles can also be output in the larger range of change in low resistance of the potentiometer. There exist the period-doubling bifurcation and the tangent bifurcation of cycle 3 in the system. The particularly interesting thing is that the bifurcation behavior has the Fengenbaum first constant in the logarithm law. Finally, the experimental circuit of the new system was designed, and the simulation of corresponding circuit was conducted.

\section{THE CIRCUIT MODELING AND ANALYSIS OF IMPROVED FOURTH-ORDER COLPITTS OSCILLATOR}

The parallel connection of a resistor $\mathrm{R}$ again at both ends of the LC parallel loop in the original fourth-order Colpitts oscillator can build an improved fourth-order Colpitts chaotic oscillator containing RLC parallel loop. The schematic diagram of circuit is as shown in Figure 1.
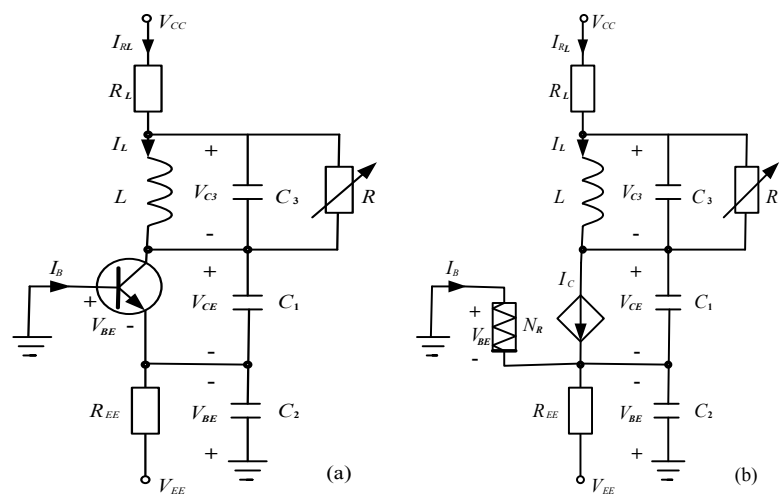

Figure 1. The circuit diagram of fourth-order robust chaotic signal source of Colpitts 
Figure 1 (a) shows that the $\mathrm{LC}_{3}$ parallel resonant circuit in the original fourth-order Colpitts oscillator becomes $\mathrm{RLC}_{3}$ parallel resonant circuit after improved. The former controls the resonant frequency $\omega 0=1 / \sqrt{L C_{3}}$ of parallel loop $\mathrm{LC}_{3}$ by changing the capacitance value of the capacitance $\mathrm{C}_{3}$, while the latter controls the oscillation frequency $\omega \mathrm{d}=1 / \sqrt{\omega_{0}^{2}-1 /\left(R C_{3}\right)^{2}}$ of parallel loop $\mathrm{RLC}_{3}$ by changing the resistance of the potentiometer $\mathrm{R}$. This is their difference. Changing the $\mathrm{R}$ has better operability and practicality than the original system changing $\mathrm{C}_{3}$ from the point of view of circuit implementation and application.

\section{A. Circuit Modeling}

The Equation (1) of the circuit system is established by Kirchhoff's law according to the equivalent schematic diagram (see Figure 1b) of improved fourth-order Colpitts oscillator:

$$
\left\{\begin{array}{l}
C_{1} \frac{d V_{C E}}{d t}=I_{R_{L}}-I_{C} \\
C_{2} \frac{d V_{B E}}{d t}=-I_{B}-I_{R_{L}}-\frac{V_{B E}+V_{E E}}{R_{E E}} \\
C_{3} \frac{d V_{C 3}}{d t}=I_{R_{L}}-I_{L}-I_{R} \\
L \frac{d I_{L}}{d t}=V_{C 3}
\end{array}\right.
$$

where:

$$
I_{R_{L}}=\frac{\left(V_{C C}-V_{C 3}-V_{C E}+V_{B E}\right)}{R_{L}}
$$

$I_{B}=F\left(V_{B E}\right)=\frac{\left(V_{B E}-V_{T H}+\left|V_{B E}-V_{T H}\right|\right)}{2 R_{O N}}$ and $I_{C}=\beta_{F} I_{B}$.

The formula above is substituted into the formula (1) to get the Formula (2) after finishing.

$$
\left\{\begin{array}{l}
C_{1} \frac{d V_{C E}}{d t}=\frac{\left(V_{C C}-V_{C 3}-V_{C E}+V_{B E}\right)}{R_{L}}-\beta_{F} F\left(V_{B E}\right) \\
C_{2} \frac{d V_{B E}}{d t}=-F\left(V_{B E}\right)-\frac{\left(V_{C C}-V_{C 3}-V_{C E}+V_{B E}\right)}{R_{L}}-\frac{V_{B E}+V_{E E}}{R_{E E}} \\
C_{3} \frac{d V_{C 3}}{d t}=\frac{\left(V_{C C}-V_{C 3}-V_{C E}+V_{B E}\right)}{R_{L}}-I_{L}-I_{R_{L}} \\
L \frac{d I_{L}}{d t}=V_{C 3}
\end{array}\right.
$$

\section{B. Equilibrium point and stability analysis}

The first order derivative of each state variable $\left(V_{C E}\right.$, $V_{B E}, V_{C 3}, I_{L}$ ) versus the time $t$ is zero for solving the equilibrium point of the system equation (2). Circuit parameters are set as: $V_{C C}=5 \mathrm{~V}, V_{E E}=-5 \mathrm{~V}, V_{T H}=0.75 \mathrm{~V}, R_{L}$ $=35 \Omega, R_{E E}=400 \Omega, R_{O N}=100 \Omega, L=100 \mathrm{mH}, C_{1}=50 \mathrm{uF}, C_{2}$ $=50 \mathrm{uF}, C_{3}=0.5 \mathrm{uF}$ and $\beta_{F}=100$. Resistance $R$ is adjustable, and the solutions are: $V_{C E}=5.4030 \mathrm{~V}, V_{B E}=0.7605 \mathrm{~V}$, $V_{C 3}=0 \mathrm{~V}$ and $I_{L}=0.0102 \mathrm{~A}$. So the equilibrium point $S=(5.4030,0.7605,0,0.0102)$.
$\mathrm{R}$ is only related to state variable $\mathrm{V}_{\mathrm{C} 3}$, and $\mathrm{V}_{\mathrm{C} 3}$ is zero. This shows that this equilibrium point has nothing to do with the resistance of the adjustable resistor $\mathrm{R}$, in other word, the equilibrium point $\mathrm{S}$ does not change with the change of the control parameter $\mathrm{R}$ of circuit. State operational orbits of improved fourth-order Colpitts oscillator disturb around this point of balance.

The Jacobian matrix (3) at the equilibrium point $\mathrm{S}$ is obtained by the system Equation (2):

$$
J_{S}=\left[\begin{array}{cccc}
\frac{-1}{R_{L} C_{1}} & \frac{1}{R_{L} C_{1}}+\frac{\beta_{F} * d F}{C_{1}} & \frac{-1}{R_{L} C_{1}} & 0 \\
\frac{1}{R_{L} C_{2}} & \frac{-1}{R_{L} C_{2}}-\frac{d F}{C_{2}}-\frac{1}{R_{E E} C_{2}} & \frac{1}{R_{L} C_{2}} & 0 \\
\frac{-1}{R_{L} C_{3}} & \frac{1}{R_{L} C_{3}} & \frac{1}{R_{L} C_{3}}-\frac{1}{R C_{3}} & \frac{-1}{C_{3}} \\
0 & 0 & \frac{1}{L} & 0
\end{array}\right]
$$

wherein: $F(V T H)=($ VBE-VTH $+\mid$ VBE - VTH $\mid) / 2 /$ $\mathrm{RON}$; $d F$ is a derivative of $\mathrm{F}(\mathrm{VTH})$ versus $\mathrm{VTH}$; $\mathrm{dF}=(1+\operatorname{sgn}(\mathrm{VBE}-\mathrm{VTH})) / 2 / \mathrm{RON}$.

The circuit parameters are substituted into the Jacobian matrix to derive the eigenvalue equation at equilibrium point $\mathrm{S}$ :

$$
\begin{aligned}
& \operatorname{det}\left|\lambda I-J_{s}\right|=0 \\
& \lambda^{3}-9 * 10^{3} \lambda^{2}+1.9 * 10^{9} \lambda-1.8 * 10^{15}=0
\end{aligned}
$$

The solved eigenvalue of solution (4) is:

$$
\begin{aligned}
& \lambda_{1}=10.1928, \lambda_{2}=1.1938, \\
& \lambda_{3}=-0.5519+1.0969 i, \lambda_{4}=-0.5519-1.0969 i
\end{aligned}
$$

$\lambda_{1}$ and $\lambda_{2}$ are greater than zero, indicating that the equilibrium point $S$ is the unstable equilibrium point.

$\lambda_{3,4}=-0.5519 \pm 1.0969 \mathrm{i}$, they are as a pair of conjugate complex roots with a negative real part, indicating that the phase trajectories of system operation disturb and form vortex rings around equilibrium points.

\section{The analysis on dynamic characteristics}

The circuit parameters of improved fourth-order Collpitts oscillator are set as: $\mathrm{V}_{\mathrm{CC}}=5 \mathrm{~V}, \mathrm{~V}_{\mathrm{EE}}=-5 \mathrm{~V}$, $\mathrm{V}_{\mathrm{TH}}=0.75 \mathrm{~V}, \mathrm{R}_{\mathrm{L}}=35 \Omega, \mathrm{R}_{\mathrm{EE}}=400 \Omega, \mathrm{R}_{\mathrm{ON}}=100 \Omega, \mathrm{L}=100 \mathrm{mH}$, $\mathrm{C}_{1}=50 \mathrm{uF}, \mathrm{C}_{2}=50 \mathrm{uF}, \mathrm{C}_{3}=0.5 \mathrm{uF}$ and $\beta_{\mathrm{F}}=100$. Resistance $\mathrm{R}$ is adjustable. Then, Lyapunov exponent spectrum and bifurcation diagram of changes in this circuit system with the control parameter $\mathrm{R}$ are calculated, as shown in Figure 3.

It can be seen from Figure 2 that the a Lyapunov exponent of improved fourth-order Collpitts oscillator always smoothly changes and is greater than zero in the two change intervals, $[1.25 \mathrm{k} \Omega 3.1 \mathrm{k} \Omega]$ and $[9 \mathrm{k} \Omega, 1 \mathrm{M} \Omega]$, of control parameter $\mathrm{R}$, indicating that the oscillator is running in the chaotic state. It should be noted that the control parameter $\mathrm{R}$ does not have period window within larger change interval, $[9 \mathrm{k} \Omega, 1 \mathrm{M} \Omega]$. Therefore, the dynamics of system when control parameters change are of better robustness. Improved fourth-order Collpitts oscillator is able to generate robust chaotic signal. 


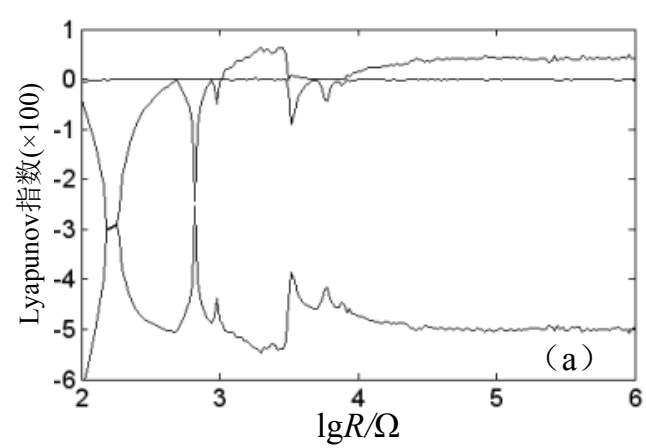

(a) Lyapunov exponent spectrum

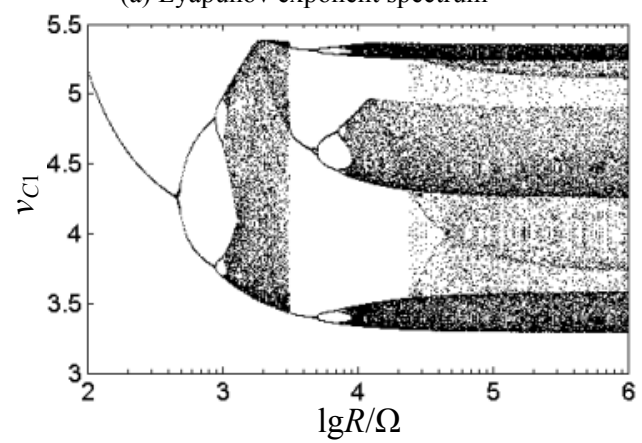

(b) Bifurcation diagram

Figure 2. The Lyapunov exponent spectrum and bifurcation diagram of changes in improved fourth-order Collpitts oscillator with adjustable potentiometer $\mathrm{R}$

Figure 2 (b) shows that the improved fourth-order Collpitts oscillator has cycle 1 , cycle 2 , cycle 3 , cycle 4 , cycle 6 and cycle 12 and other periodic states which appear in turn besides two robust chaotic regions identified by the above Lyapunov exponent spectrum in the change range from $100 \Omega$ to $1 \mathrm{M}$ of control parameter resistance $\mathrm{R}$. The corresponding relationship between control parameter $\mathrm{R}$ and the periodic running state of the system is shown in Table 1.

TABLE I.

TYPE SIZES FOR CAMERA-READY PAPERS

\begin{tabular}{|c|c|c|c|}
\hline $\mathrm{R} / \Omega$ & $\begin{array}{c}\text { Number of } \\
\text { cycles }\end{array}$ & Signal characteristics & Phase trajectory \\
\hline $100 \sim 450$ & 1 & Sinusoidal oscillation & see Figure 3 (a) \\
\hline $450 \sim 870$ & 2 & Cycle 2 oscillation & see Figure 3 (b) \\
\hline $870 \sim 1005$ & 4 & Cycle 4 oscillation & see Figure 3 (c) \\
\hline $3.2 \mathrm{k} \sim 5 \mathrm{k}$ & 3 & Cycle 3 oscillation & see Figure 3 (d) \\
\hline $5 \mathrm{k} \sim 7.2 \mathrm{k}$ & 6 & Cycle 6 oscillation & see Figure 3 (e) \\
\hline $7.2 \mathrm{k} \sim 8.1 \mathrm{k}$ & 12 & Cycle 12 oscillation & see Figure 3 (f) \\
\hline $\begin{array}{c}1.25 \mathrm{k} \sim 3.1 \mathrm{k}, \\
9 \mathrm{k} \sim 1 \mathrm{M}\end{array}$ & no & Chaotic state & see Figure 4 \\
\hline
\end{tabular}

The bifurcation diagram of the improved fourth-order Collpitts oscillator with $\mathrm{R}$ value of approximate $450 \Omega$ reveals the first period-doubling bifurcation occurring in system which means that single cycle is split into 2 cycles. The second period-doubling bifurcation occurs again in the system when $\mathrm{R}$ value is approximately $870 \Omega$, thereby, 2 cycles are split into 4 cycles. The third period-doubling bifurcation continues to occur in the system, while the system gradually enters into the first chaotic region from period-doubling bifurcation, with the $R$ value of about $1010 \Omega$. Tangent bifurcation of cycle 3 arises in the system, with the control parameter $\mathrm{R}$ value of approximate $3120 \Omega$, and then it enters into the second chaotic region after the phenomenon of chaotic crisis led to by the period-doubling bifurcation. Figure 2 (b) shows that bifurcation behaviors arising with the changes of control parameter $\mathrm{R}$ have the obvious self-similar structure.

The ratio of the control parameter interval lengths of two successive bifurcations has a characteristic of equal proportion according to the calculating formula of the Feigenbaum first constant. The ratio eventually approaches a constant 4.6692...with the increase of bifurcation frequency. Data in Figure 3 (b) are substituted into the calculation formula of Feigenbaum first constant: $\delta=\lim _{n \rightarrow \infty} \frac{k_{n}-k_{n-1}}{k_{n+1}-k_{n}}$, to get $(870-450) /(1005-870)=3.111 \ldots$, which is a far cry from the Feigenbaum first constant. Interestingly, if the logarithm of value corresponding to the control parameter of bifurcation is taken to calculate Feigenbaum first constant: $(\log 870-\log 450) /(\log 1005$ $\log 870)=4.566 \ldots$, then the result of this calculation is very close to the Feigenbaum first constant value. Whether it indicates that the bifurcation in some of non-linear systems meets the Feigenbaum first constant of logarithmic form needs to be further investigated.

Figure 3 shows multiple typical phase trajectories of period windows. Therefore, improved Colpitts oscillator can run in a single or multiple cycle state and thus output single or multiple cycle signal under the control of the system control parameter R. It can also make the system enter into a chaotic state through different bifurcation forms, such as period-doubling bifurcation or periodtripling bifurcation.

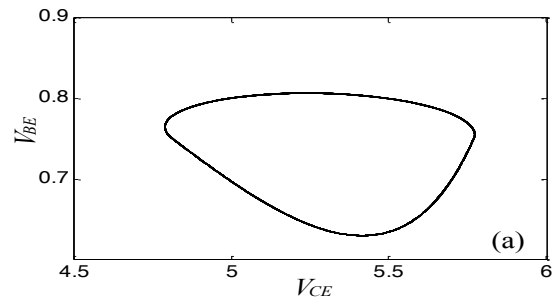

(a) $\mathrm{R}=100 \Omega$, the phase trajectory of VCE-VBE plane (cycle 1)

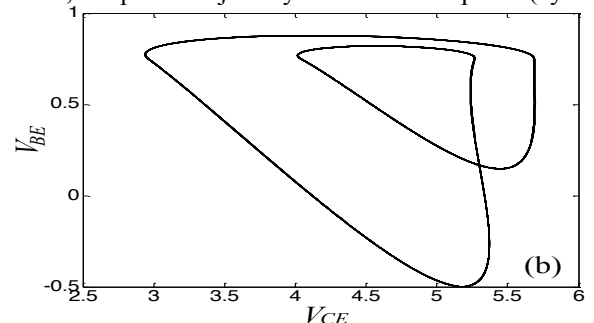

(b) $\mathrm{R}=600 \Omega$, the phase trajectory of VCE-VBE plane (cycle 2)

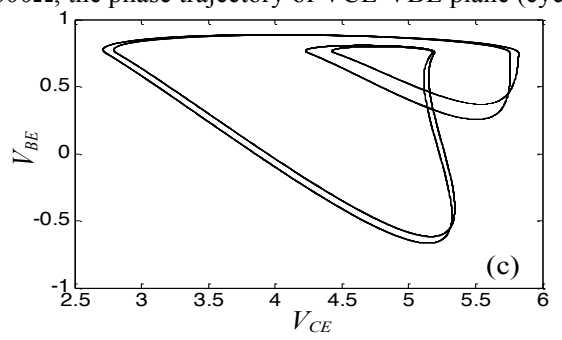

(c) $\mathrm{R}=900 \Omega$, the phase trajectory of VCE-VBE plane (cycle 4) 
PAPER

SIMULATION OF IMPROVED FOURTH-ORDERCOLPITTS OSCILLATOR

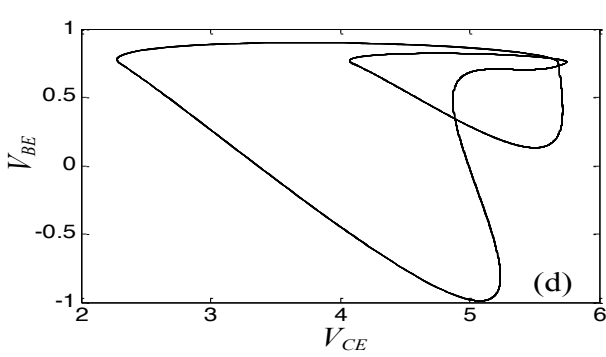

(d) $\mathrm{R}=4 \mathrm{k} \Omega$, the phase trajectory of VCE-VBE plane (cycle 3)

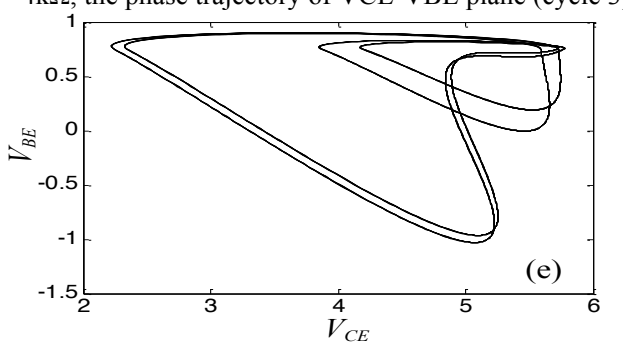

(e) $\mathrm{R}=6 \mathrm{k} \Omega$, the phase trajectory of VCE-VBE plane (cycle 6)

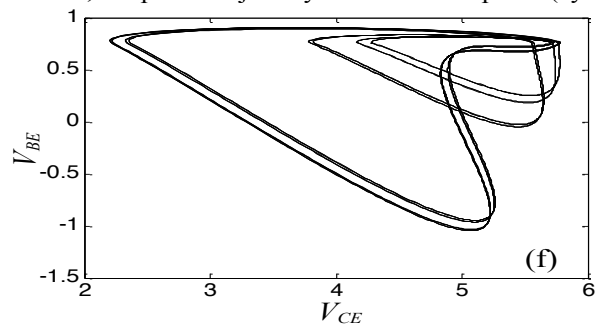

(f) $\mathrm{R}=7.5 \mathrm{k} \Omega$, the phase trajectory of VCE-VBE plane (cycle 12)

Figure 3. The phase trajectories of multi-cycle signals generated by improved fourth-order Colpitts oscillator

Figure 4 shows the phase trajectories of a set of voltage and current variables output by the improved Colpitts oscillator with the control parameter $\mathrm{R}$ of $200 \mathrm{k} \Omega$. The comparison of using $\mathrm{R}$ as the system control parameter with the original fourth-order system with capacitance $\mathrm{C}_{3}$ controlling the operation state of system shows that the former circuit is more convenient to implement, and the changing ranges of control parameters are wider, thus ensuring the operation of the system being more stable.

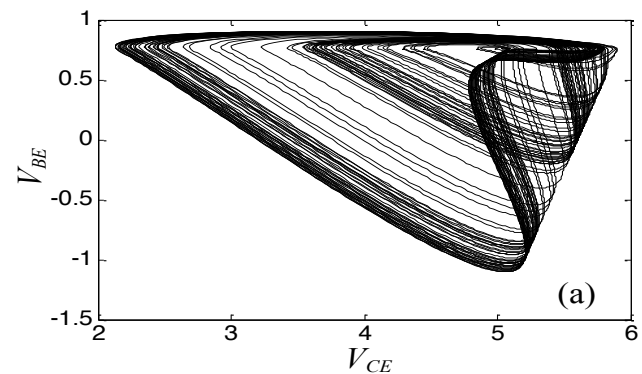

(a) The phase trajectory of VCE-VBE plane

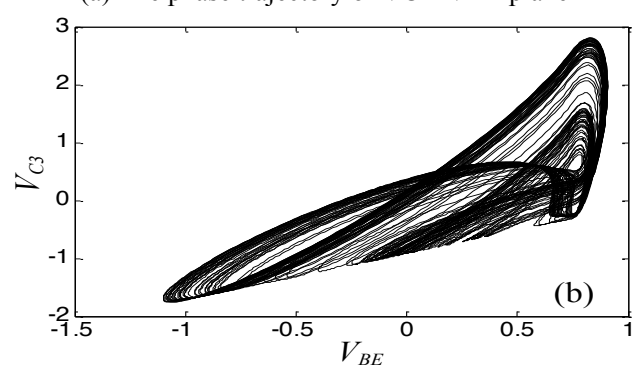

(b) The phase trajectory of VBE-VC3 plane

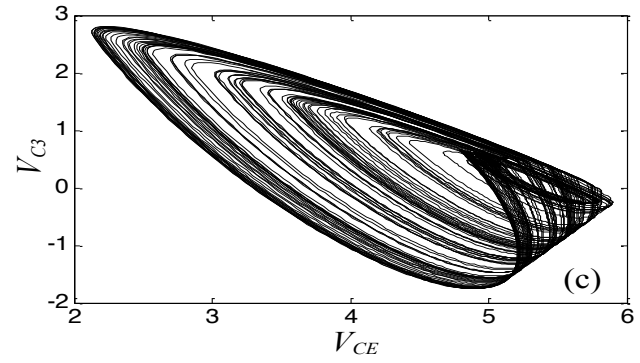

(c) The phase trajectory of VBE-VC3 plane

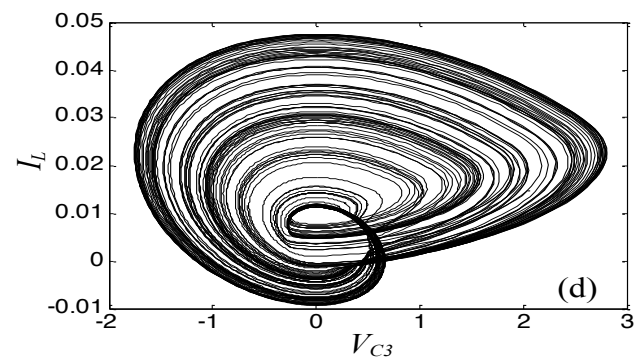

(d) The phase trajectory of VC3- IL plane

Figure 4. $\mathrm{R}=200 \mathrm{k} \Omega$ the phase trajectories of improved fourth-order Colpitts oscillator

\section{EXPERIMENTAL VERIFICATIONS OF CIRCUIT}

\section{A. Circuit simulation}

The experimental verification of circuit simulation is an effective mean to study the dynamics of nonlinear system which is of a great significance for judging whether the newly constructed chaotic system has application value. The experimental circuit is built by Multisim circuit simulation software according to Figure 1. The values of circuit parameters are: $V_{C C}=5 \mathrm{~V}, V_{E E}=5 \mathrm{~V}, V_{T H}=0.75 \mathrm{~V}$, $R_{L}=35 \Omega, R_{E E}=400 \Omega, R_{O N}=100 \Omega, L=100 \mathrm{mH}, C_{1}=50 \mathrm{uF}$, $C_{2}=50 \mathrm{uF}, C_{3}=0.5 \mathrm{uF}$ and $\beta_{F}=100$. The resistance $R$ is adjustable. The strange attractors of chaotic oscillator of improved fourth-order Colpitts are observed by oscilloscope, as shown in Figure 5.

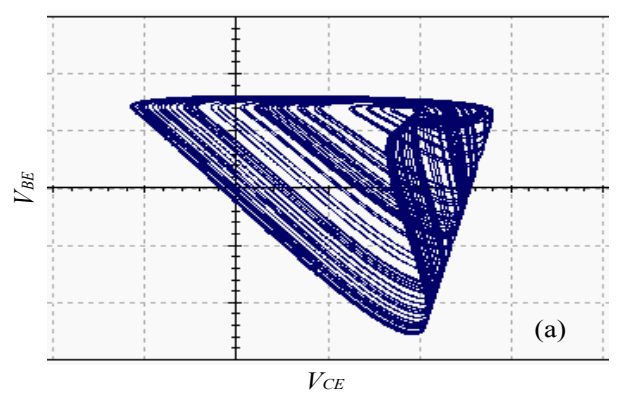

(a) $1 \mathrm{v} / \operatorname{div},-3 ; 0.5 \mathrm{v} / \operatorname{div}, 0$

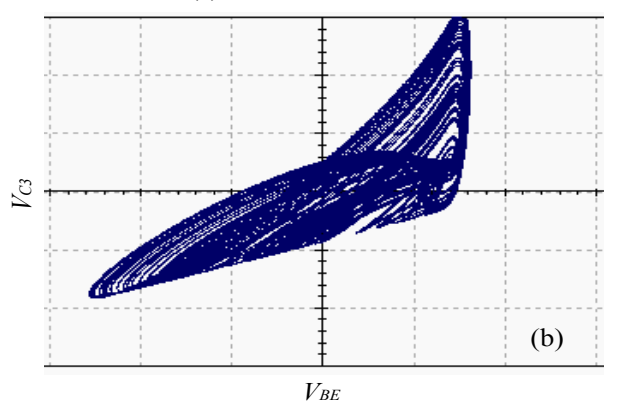

(b) $0.5 \mathrm{~V} / \mathrm{div}, 0 ; 1 \mathrm{v} / \mathrm{div}, 0$ 


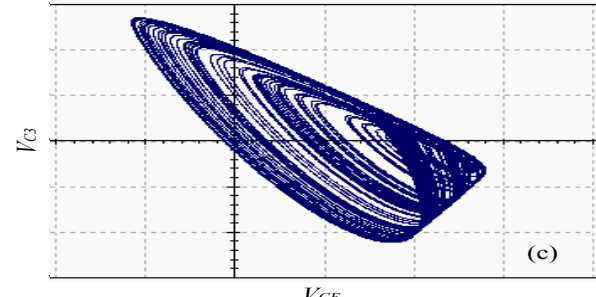

(c) $1 \mathrm{~V} / \mathrm{div},-3 ; 1 \mathrm{v} / \mathrm{div},-0.4$

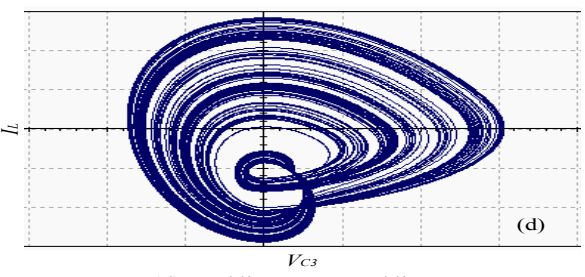

(d) $1 \mathrm{~V} / \mathrm{div}, 0 ; 10 \mathrm{~mA} / \mathrm{div},-2$

Figure 5. $\mathrm{R}=200 \mathrm{k} \Omega$ the circuit simulation phase trajectories of improved fourth-order Colpitts oscillator

The chaotic attractors of improved fourth-order Colpitts oscillator of visible numerical calculation in Figure 4 and the chaotic attractors output by circuit simulation in Figure 5 with the same parameters have a good consistency.

\section{B. Circuit experiment}

The experimental circuit is composed by the non-linear elements: transistors, and passive components, such as resistances, capacitances and inductors. The circuit structure is simple. Transistor 2N3904 is used (or may choose transistor, $2 \mathrm{~N} 2222$ or $\mathrm{BC} 108$, with similar characteristics as substitution). The $100 \mathrm{mH}$ inductance can use copper wire with thick diameter wound on the magnetic body by itself to reduce its internal resistance. The remaining component parameters and power are the same with the simulation circuit parameters. Improved fourth-order Colpitts oscillator outputs the cycles or chaotic phase trajectories, as shown in Figure 6, which were observed by Tektronix digital oscilloscope. The comparisons of Figure 6 (a) to Figure 3 (b) and Figure 6 (b) to Figure 5 (a) show that the results of the circuit experiment and the above numerical simulation or circuit simulation have a better consistency.

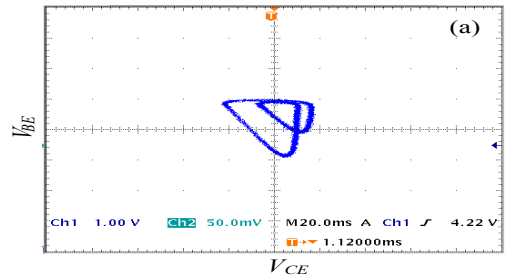

(a) $\mathrm{R}=600 \Omega$, the phase trajectory of VCE-VBE plane (cycle 2)

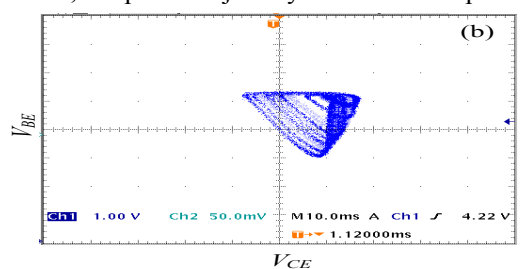

(b) $\mathrm{R}=200 \mathrm{k} \Omega$, the phase trajectory of VCE-VBE plane (chaotic attractor)

Figure 6. The phase trajectories output by the circuit experiment of improved fourth-order Colpitts oscillator

\section{CONCLUSIONS}

An improved fourth-order Colpitts oscillator containing $R L C$ parallel resonance circuit was proposed in this study. The stabilities of the equilibrium points were analyzed, and the numerical calculations of Lyapunov exponent spectrum, bifurcation diagram and the phase trajectories of chaotic attractors of changes with adjustable potentiometer $R$ were done in the new system on the basis of circuit modeling. The analysis shows that the new system has the period-doubling bifurcation, tangent bifurcation and other nonlinear dynamic behaviors. Interestingly, it is found that the bifurcation law of the system has the Feigenbaum first constant in the logarithm law. The experimental circuit of the new system was designed, and the experimental results and theoretical analysis have a good consistency. Since the new system uses the resistance of potentiometer as a control parameter, its circuit implementation is more convenient than that of the original fourth-order Colpitts oscillator with capacitance as a control parameter. The Lyapunov exponent spectrum indicates that chaotic signals generated by the new system have better robustness with control parameters in a larger range of change.

\section{REFERENCES}

[1] G. Eason, B. Noble, and I. N. Sneddon, "On certain integrals of Lipschitz-Hankel type involving products of Bessel functions," Phil. Trans. Roy. Soc. London, vol. A247, pp. 529-551, April 1955.

[2] J. Clerk Maxwell, A Treatise on Electricity and Magnetism, $3^{\text {rd }}$ ed., vol. 2. Oxford: Clarendon, 1892, pp.68-73.

[3] I. S. Jacobs and C. P. Bean, "Fine particles, thin films and exchange anisotropy," in Magnetism, vol. III, G. T. Rado and H. Suhl, Eds. New York: Academic, 1963, pp. 271-350.

[4] K. Elissa, "Title of paper if known," unpublished.

[5] R. Nicole, "Title of paper with only first word capitalized", $J$. Name Stand. Abbrev., in press.

[6] Y. Yorozu, M. Hirano, K. Oka, and Y. Tagawa, "Electron spectroscopy studies on magneto-optical media and plastic substrate interface," IEEE Transl. J. Magn. Japan, vol. 2, pp. 740741, August 1987 [Digests $9^{\text {th }}$ Annual Conf. Magnetics Japan, p. 301, 1982]. http://dx.doi.org/10.1109/TJMJ.1987.4549593

[7] M. Young, The Technical Writer's Handbook. Mill Valley, CA: University Science, 1989.

\section{AUTHORS}

Qiao Xiaohua is a professor in Jiangsu University of Technology (School of Electrical and Information Engineering). His research interests include nonlinear circuits and system and information dynamic system research work.

Sun Yuxia is an associate professor in Jiangsu University of Technology (School of Electrical and Information Engineering). Her research interests include design and simulation of nonlinear electronic circuits and system.

This work was supported in part by the Natural Science Foundation of Jiangsu Province (Grant No.2012583). Received 09 April 2013. Published as resubmitted by the authors 12 June 2013. 